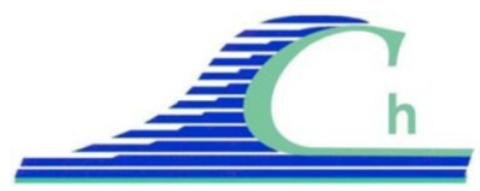

XII ${ }^{\text {ìmes }}$ Journées Nationales Génie Côtier - Génie Civil

Cherbourg, 12-14 juin 2012

DOI:10.5150/jngcgc.2012.010-K C Editions Paralia CFL

disponible en ligne - http://www.paralia.fr - available online

\title{
Modélisation hydrodynamique tridimensionnelle en coordonnées curvilignes non-orthogonales de l'estuaire de la Seine
}

\section{Youen KERVELLA ${ }^{1,2}$, Iman KHOJASTEH POUR FARD ${ }^{1}$, Pierre LE HIR ${ }^{1}$, Emmanuel RENAULT ${ }^{1}$, Jean-Philippe LEMOINE ${ }^{3}$}

1. IFREMER, DYNECO/PHYSED, Centre de Brest, 29280 Plouzané, France.

2. OPEN OCEAN, Pépinière Créatic, 115 rue Claude CHAPPE, Technopole Brest Iroise, Site de la Pointe du Diable, 29280 Plouzane, France. www.openocean.fr youen.kervella@openocean.fr

3. GIP Seine-Aval, Rouen, France.

\section{Résumé :}

Dans le cadre du projet MODEL mené par le laboratoire DYNECO/PHYSED de l'Ifremer et financé par le Groupement d’Intérêt Public Seine-Aval (GIPSA, http://seine-aval.crihan.fr), un modèle régional à coordonnées curvilignes nonorthogonales a été mis en place sur la baie de Seine et la Seine. Ce modèle est basé sur le code tridimensionnel MARS3D (LAZURE \& DUMAS, 2008) dont les équations ont été modifiées afin de prendre en compte la déformation des mailles par rapport à un maillage cartésien régulier (GOURRION, 2005). La validation hydrodynamique du modèle est réalisée par comparaison à des mesures marégraphiques (Grand Port Maritime de Rouen) de Honfleur jusqu’à Poses, la limite de marée dynamique de la Seine, par comparaison à des mesures de profils de courants obtenus par ADCP immergés (IXSurvey, GPM Rouen) et par comparaison à des mesures de salinité (bouée MAREL-Honfleur). Les résultats sont très satisfaisants dans tout l'estuaire, le modèle curviligne permettant de pallier le manque d'efficacité des modèles cartésiens dans la partie la plus amont (difficulté à représenter correctement les sections d'écoulement et leur variabilité au cours de la marée (WAELES, 2005)).

Des comparaisons entre les cartographies de salinité de surface du modèle à différents instants et des images satellites sont également présentées et montrent une bonne adéquation au niveau de la géométrie des structures de surface de l'embouchure de la Seine.

Mots-clés :

Modélisation régionale - Maillage curviligne - Hydrodynamique - Salinité - Estuaire de la Seine

\section{Introduction}

Un modèle morphosédimentaire de l'estuaire de la Seine a été précédemment développé dans le cadre des programmes Seine-Aval I, II et III (Waeles, 2005 ; Waeles, 2008). 
Dans le cadre du nouveau programme de recherche MODEL de Seine-Aval, il a été mis en évidence la nécessité de raffiner le maillage du modèle Seine-Aval, en particulier pour accroître la pertinence des réponses morphodynamiques du système estuaire et embouchure de Seine, et ce faisant l'opportunité de basculer sur la plateforme de modélisation MARS, qui bénéficie à Ifremer d'un effort de parallélisation qui permet des performances de calcul nettement meilleures.

Les caractéristiques essentielles du nouveau modèle mis en place sont les suivantes :

- code de base MARS3D à la place de SiAM concernant la courantologie.

- mise en place d'une nouvelle grille de calcul curvilinéaire,

- semi-couplage avec un modèle de vagues WaveWatch III sur grille non structurée,

- nouveau module de calcul des évolutions sédimentaires (Le Hir et al., 2011),

- ensemble des codes et modules de calcul programmés en calcul parallèle.

Après une brève description de la création et du paramétrage de la version hydrodynamique de ce modèle, cet article analyse la confrontation de quelques résultats à des mesures récentes. Des comparaisons entre des cartographies du panache de salinité de la Seine issues du modèle et des images satellites sont ensuite présentées et discutées.

\section{Matériel et méthodes}

La démarche adoptée dans notre cas est de réaliser un maillage curviligne structuré nonorthogonal en 2D de la baie de Seine et de la rivière jusqu'à Poses (limite de marée dynamique), en suivant le plus possible le trait de côte et en adaptant les tailles de mailles aux vitesses de courant susceptibles d'être rencontrées, afin de maintenir un pas de temps de calcul correct par la suite (formulé selon le critère CFL : $u<\Delta x / \Delta t$ et $v<\Delta y / \Delta t)$.

L'intérêt de réaliser ce type de maillage est d'une part d'orienter les lignes de maillage dans les directions privilégiées de l'écoulement et d'autre part de pouvoir raffiner les mailles à certains endroits (comme l'embouchure de la Seine, la baie des Veys, la zone littorale du Calvados, ...) sans pour autant affecter l'ensemble du domaine d'étude et trop augmenter le temps de calcul. Les fleuves et leurs méandres peuvent aussi être représentés en 3 dimensions et il est ainsi possible d'étudier les échanges, notamment en termes de dynamique sédimentaire, entre les compartiments "chenal" et "berges".

L'emprise du maillage couvre toute la baie de Seine, de Cherbourg à Fécamp, et la Seine jusqu'à Poses (PK 200). Le maillage compte (en 2D) 79213 mailles dont 20821 mailles en eau (mailles pour lesquelles les calculs hydrodynamiques sont effectués). Les tailles de mailles sont variables (rappelons qu'en curviligne non-orthogonal, les mailles sont des quadrilatères quelconques et ne sont donc pas forcément rectangles) avec des dimensions maximales de $2000 \mathrm{~m} \times 2500 \mathrm{~m}$ près de la frontière du modèle (nord de la baie de Seine) et des dimensions minimales de $80 \mathrm{~m} \times 150 \mathrm{~m}$ sur notre principale zone d'intérêt : l'embouchure de la Seine. 


\section{XII ${ }^{\text {èmes }}$ Journées Nationales Génie Côtier - Génie Civil \\ Cherbourg, 12-14 juin 2012}

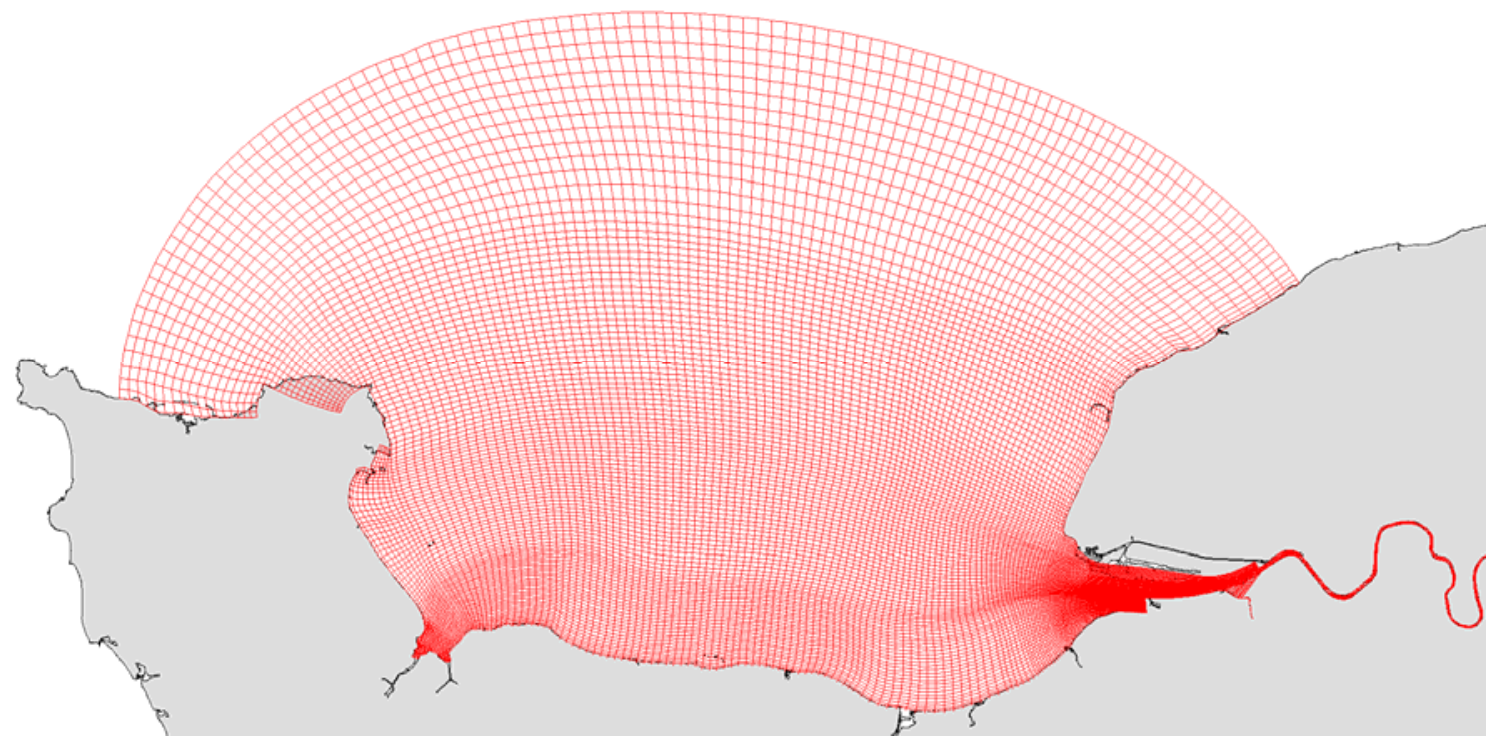

Figure 1. Maillage curviligne de la baie de Seine.

La figure 1 présente le maillage dans son intégralité : une emprise de la baie de Cherbourg à Fécamp avec une frontière incurvée permettant la quasi-orthogonalité des mailles avec la côte à l'ouest et à l'est de la baie ; des mailles volontairement agrandies près de cette frontière afin de limiter le temps de calcul, et des mailles très fines en baie des Veys (environ $200 \mathrm{~m} \times 200 \mathrm{~m}$ ) et à l'embouchure de la Seine. Les mailles suivent le trait de côte (dimension des mailles le long de la côte du Calvados : environ $500 \mathrm{~m} \times$ $300 \mathrm{~m}$, le critère définissant cette dimension minimale étant essentiellement le temps de calcul du modèle de vagues) et sont orientées dans le sens des principaux courants côtiers (parallèles à la côte). Dans le secteur de l'embouchure, le maillage suit autant que possible la bathymétrie du chenal de navigation, délimité plus en amont par les digues submersibles, ce qui permet également d'orienter les mailles dans le sens des maximas de courants de marée sur cette zone et donc de réduire le temps de calcul (pour un même pas de temps, la satisfaction du critère CFL conduit à allonger les mailles dans le sens du courant principal, ici le courant de marée. Néanmoins, il convient de prendre aussi en compte la possibilité d'occurrence de courants transversaux engendrés par le vent, notamment dans le secteur de l'embouchure).

Le maillage de la section fluviale comporte, d'amont en aval, de 3 à 8 mailles dans sa dimension transversale, avec une largeur de maille de l'ordre de $100 \mathrm{~m}$ et plus de 500 mailles dans sa dimension longitudinale (de Honfleur à Poses) avec une longueur de maille de l'ordre de 300 m. Les îles en amont de Rouen sont également prises en compte.

La bathymétrie (ainsi que la structure 2D des niveaux moyens de la mer, telle que fournie par le SHOM) de notre modèle est reconstituée à l'aide du logiciel BMGTOOLS 
développé sous la direction de l'Ifremer, dont les routines d'interpolation et de visualisation ont été modifiées pour prendre en compte la curvilinéarité du maillage. La matrice des points du maillage est une matrice incomplète ; il faut donc la compléter avec des points fictifs afin d'obtenir une grille structurée. Ces points fictifs doivent être ignorés lors de l'interpolation de la bathymétrie. Les données bathymétriques les plus récentes et les plus précises existant dans la zone ont été utilisées :

- Pour la baie de Seine : MNT (Modèle Numérique de Terrain) avec un pas de $100 \mathrm{~m}$ de la Bretagne Nord et MNT avec un pas de $100 \mathrm{~m}$ en Baie de Seine (source: IFREMER/SHOM)

- Pour l'embouchure de la Seine : des données du GPM Rouen de décembre 2009 sous forme de grille de $10 \mathrm{~m} \times 10 \mathrm{~m}$ pour l'embouchure jusqu'à Tancarville (source : GIP Seine-Aval)

- Pour la Seine: des données de 2008 sous forme de grille de $5 \mathrm{~m} \times 5 \mathrm{~m}$ entre Tancarville et Rouen (source : GPM Rouen, GIP Seine-Aval) et des données de 2005 sous forme de grille de $1 \mathrm{~m} \times 1 \mathrm{~m}$ acquises à l'aide de sondeurs mono- et multifaisceaux entre Rouen et Poses (source : GPM Rouen, GIP Seine-Aval).

La bathymétrie est ensuite incorporée au modèle hydrodynamique MARS3D (LAZURE \& DUMAS, 2008), qui est ici utilisé dans sa version tridimensionnelle avec 10 niveaux sigma équi-répartis sur la verticale. Les équations du modèle ont été modifiées afin de prendre en compte le caractère curviligne du maillage (GOURRION, 2005): des opérateurs définissant la déformation de chaque maille, en termes de taille et d'orientation par rapport à un repère cartésien fixe, sont calculés et incorporés dans les équations du modèle.

Le modèle est forcé à sa limite nord par les niveaux d'eau issu du modèle réajusté CSTFrance (SHOM) et par un apport d'eau douce au niveau du barrage de Poses en respectant le débit réel de la Seine (données GPM Rouen).

En ce qui concerne les forçages atmosphériques, les sorties du modèle ARPEGE de Météo-France (15 km de résolution) sont utilisées.

Afin d'obtenir une bonne représentation de la marée et sa propagation dans l'estuaire, la paramètre des frottements sur le fond a été calibré (la géométrie étant déjà fixée par la bathymétrie) pour dissiper plus ou moins l'énergie de l'onde de marée et par conséquent faire varier son amplitude.

Ainsi, pour l'embouchure, une distribution de la longueur de rugosité définissant les frottements sur le fond a été établie sur la base d'une cartographie des faciès sédimentaires (LESOURD, 2000 ; LEMOINE \& GUILLERME, 2010): une valeur (réaliste) de $10 \mathrm{~mm}$ a été affectée aux galets et graviers, $5 \mathrm{~mm}$ aux sables grossiers et moyens, $2 \mathrm{~mm}$ aux sables fins, jusqu'à atteindre $0.1 \mathrm{~mm}$ pour les vases.

L'estuaire fluvial a ensuite été divisé en plusieurs parties présentant des longueurs de rugosité différentes mais progressives afin de caler au mieux le signal de marée du 


\section{XII ${ }^{\text {èmes }}$ Journées Nationales Génie Côtier - Génie Civil \\ Cherbourg, 12-14 juin 2012}

modèle par rapport aux données des marégraphes situés le long du fleuve (données GPM Rouen).

Les résultats du modèle ainsi calibré sont présentés dans la section suivante.

\section{Résultats}

\subsection{Elévation de la surface libre}

Les résultats d'élévation de surface libre obtenus dans la Baie de Seine sont comparés en plusieurs points de la Baie de Seine aux données du fichier CST France, élaboré par le SHOM à partir de résultats de modèles de propagation de marée et de mesures marégraphiques. Ces comparaisons sont très satisfaisantes et ne sont pas présentées dans cet article orienté sur l'estuaire.

Pour la partie estuarienne, la validation a été effectuée par comparaison à des mesures marégraphiques (fourniture GIPSA et GPM Rouen) réalisées en 16 points de la Seine toutes les 5 minutes. Pour plus de clarté et afin de mettre en évidence l'amortissement de l'onde de marée de l'aval à l'amont de l'estuaire, 7 points de comparaison sont présentés (figure 2) depuis Honfleur, à l'embouchure de la Seine, jusqu'à Pont de l'Arche, le marégraphe le plus en amont, près de la limite de marée dynamique (barrage de Poses). La distance séparant Honfleur de Pont de l'Arche est de $200 \mathrm{~km}$.

Les résultats sont présentés pour une période de 10 jours (du 16/04/2007 au 26/04/2007) sous des conditions moyennes de débit (environ $450 \mathrm{~m}^{3} \mathrm{~s}^{-1}$ ) et en prenant en compte dans les simulations le forçage exercé par le vent (modèle ARPEGE de Météo-France).

Ces résultats sont globalement très satisfaisants malgré des différences de hauteurs d'eau de quelques centimètres notamment lors de la transition entre les phases de viveeau et morte-eau (fin de la période présentée). La partie la plus amont de la rivière qui est plus difficile à simuler car l'énergie de l'onde de marée est très dissipée par le frottement sur le fond, est très bien représentée. Ces résultats sont certainement liés à l'utilisation de mailles curvilignes qui permet d'obtenir une géométrie "réaliste" de la rivière et de prendre en compte ses méandres ainsi que les îles et les bras morts, et qui permet de pallier au manque d'efficacité (sections de l'écoulement difficiles à décrire précisément) des modèles classiques (cartésiens) dans cette partie amont de la Seine (WAELES, 2005).

Afin de vérifier la robustesse du modèle, les mêmes comparaisons sont réalisées pour une période d'étiage de la Seine (débit d'environ $200 \mathrm{~m}^{3} \mathrm{~s}^{-1}$ ) et pour une période de crue très importante (débit supérieur à $2200 \mathrm{~m}^{3} \mathrm{~s}^{-1}$ ). Dans ces conditions, les variations de la surface et du niveau moyen de la Seine sont également très bien représentés. 

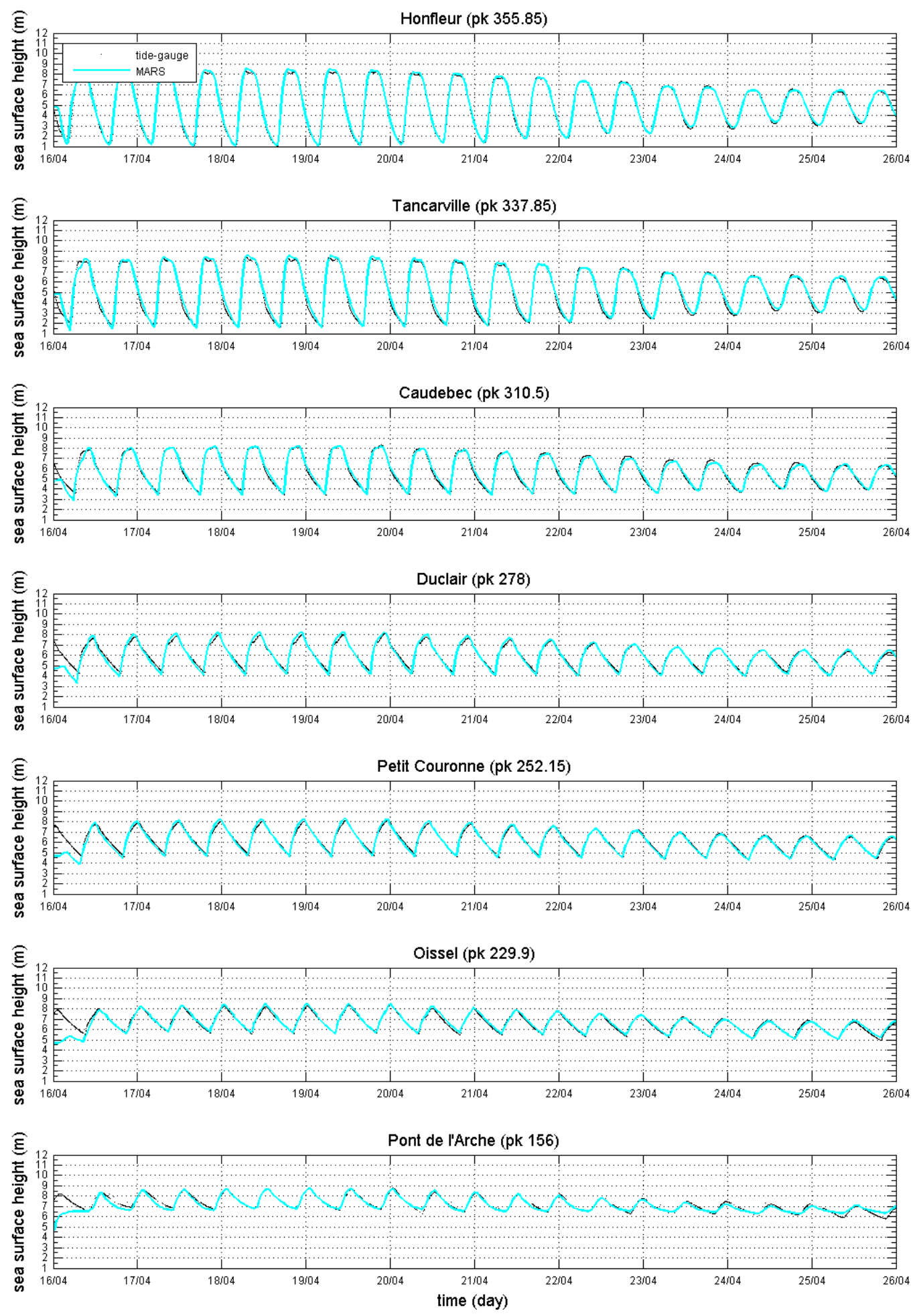

Figure 2. Comparaisons d'élévations de la surface libre dans l'estuaire (points dans l'ordre de l'aval à l'amont) par débit moyen (environ $450 \mathrm{~m}^{3} \mathrm{~s}^{-1}$ ). Mesures marégraphiques en noir et modèle MARS3D en bleu clair. 


\section{XII ${ }^{\text {èmes }}$ Journées Nationales Génie Côtier - Génie Civil \\ Cherbourg, 12-14 juin 2012}

\subsection{Vitesses}

La capacité du modèle à reproduire les intensités et les directions des vitesses de courant est évaluée grâce à une campagne de mesures réalisée en 2004 par la société IXSURVEY pour le Grand Port Maritime de Rouen. Toutes les mesures sont réalisées à l'aide d'ADCP (Acoustic Doppler Current Profiler) posés sur le fond qui fournissent des profils de courants sur toute la colonne d'eau au cours du temps. Les points de mesures [respectivement : La Risle (pt. 1), Courval (pt. 2), la Brèche du Ratier (pt. 3), Cokburn (pt. 4) et le Ratier sud (pt. 5)] sont indiqués sur la figure 3.

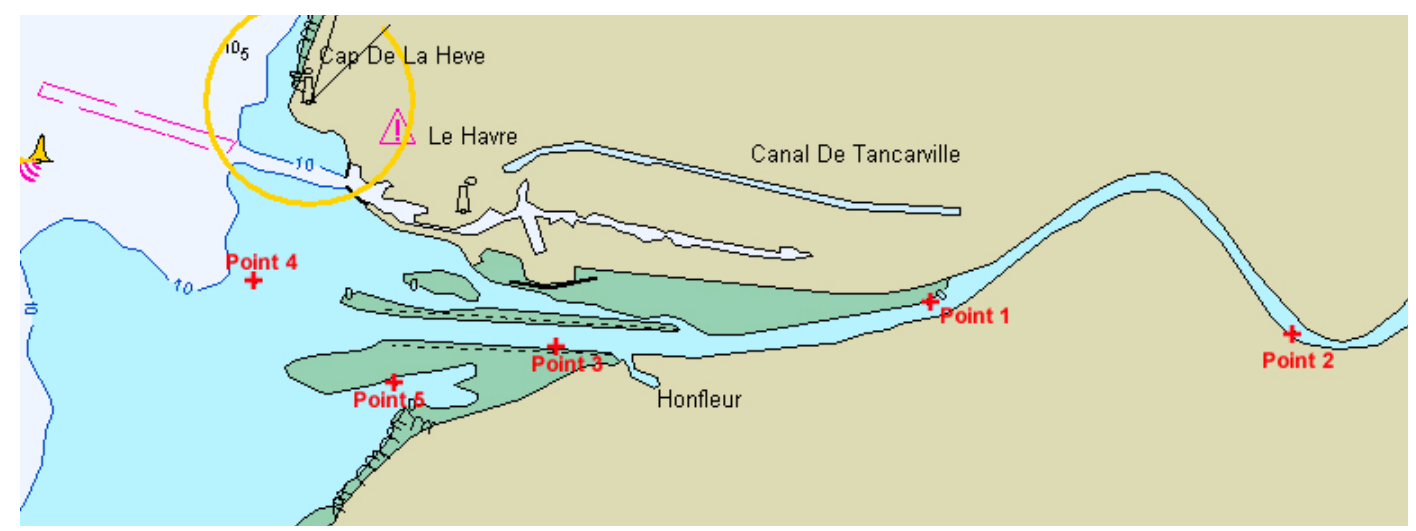

Figure 3. Localisation des points de mesures du courant (IXSURVEY, 2004).

Les figures 4 et 5 présentent les comparaisons entre ces mesures ADCP et le modèle MARS3D curviligne à Courval (point 2 de la figure 3), respectivement en termes d'intensité de vitesse et de direction. Ces figures sont divisées en 3 parties: comparaison des vitesses (respectivement directions) moyennées sur la colonne d'eau issues de l'ADCP et du modèle (en haut), vitesse (respectivement direction) sur la colonne d'eau simulée (au milieu) et vitesse (respectivement direction) sur la colonne d'eau enregistrée par l'ADCP (en bas).

Le point Courval se situe dans le premier méandre du fleuve. Les vitesses calculées par le modèle atteignent jusqu'à $2 \mathrm{~m} \mathrm{~s}^{-1}$ en fin de jusant, et correspondent ainsi aux intensités mesurées, avec une légère sous-estimation globale. Les structures du courant sur la colonne d'eau sont correctement reproduites au cours du temps (extrema de valeurs du courant bien placés et possédant la bonne intensité). Les étales de pleine mer et de basse mer simulés par le modèle semblent être un peu plus longs que ceux mesurés. Les changements de direction (renverses de courant) s'opèrent de manière identique sur toute la colonne d'eau. Ils correspondent parfaitement aux observations.

Par faute de place, les comparaisons aux autres points ne sont pas présentés dans cet article mais révèlent également des résultats très satisfaisants. 

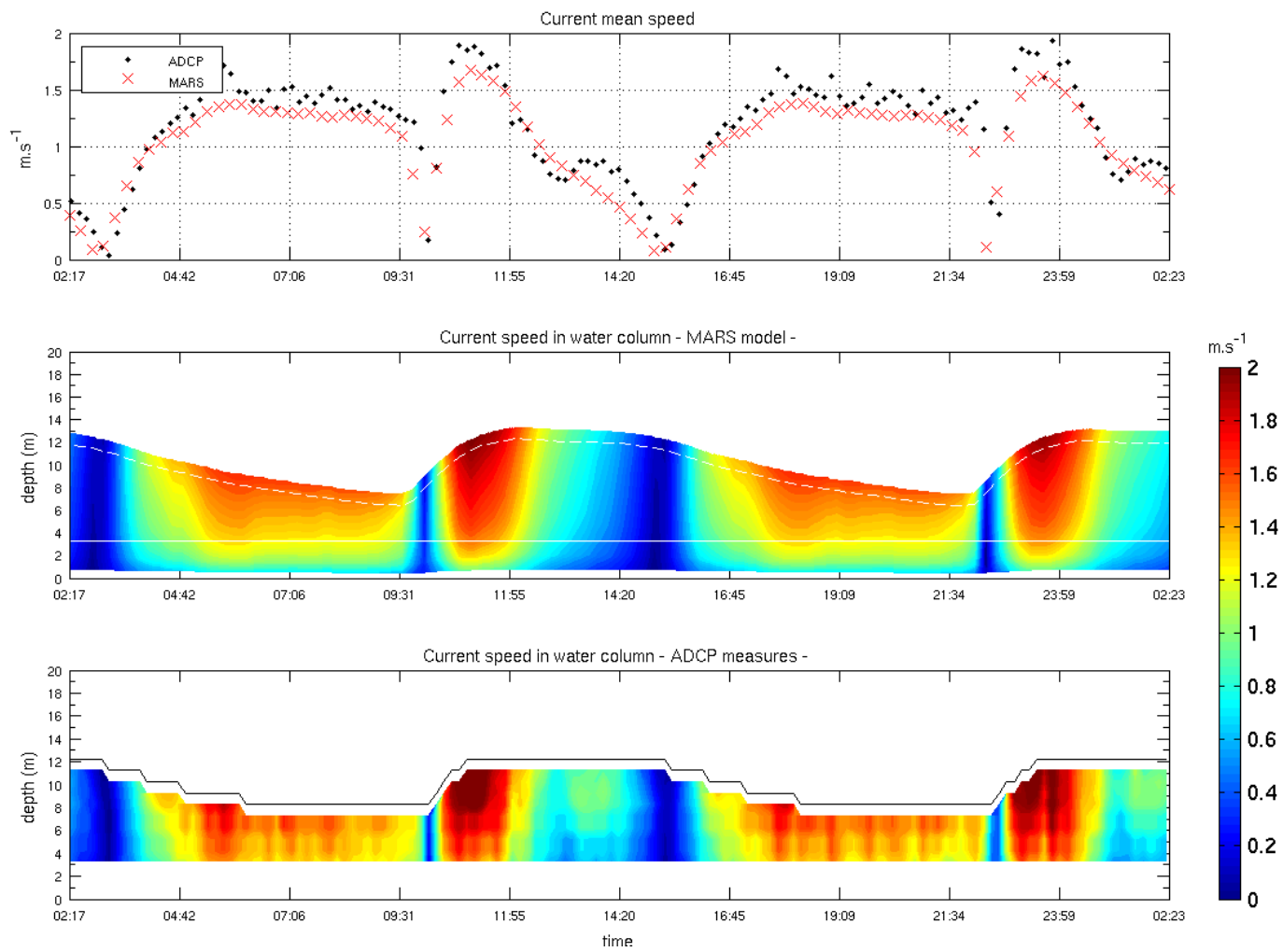

Figure 4. Intensité du courant à Courval (point 2) du 13/11/2004 au 14/11/2004.
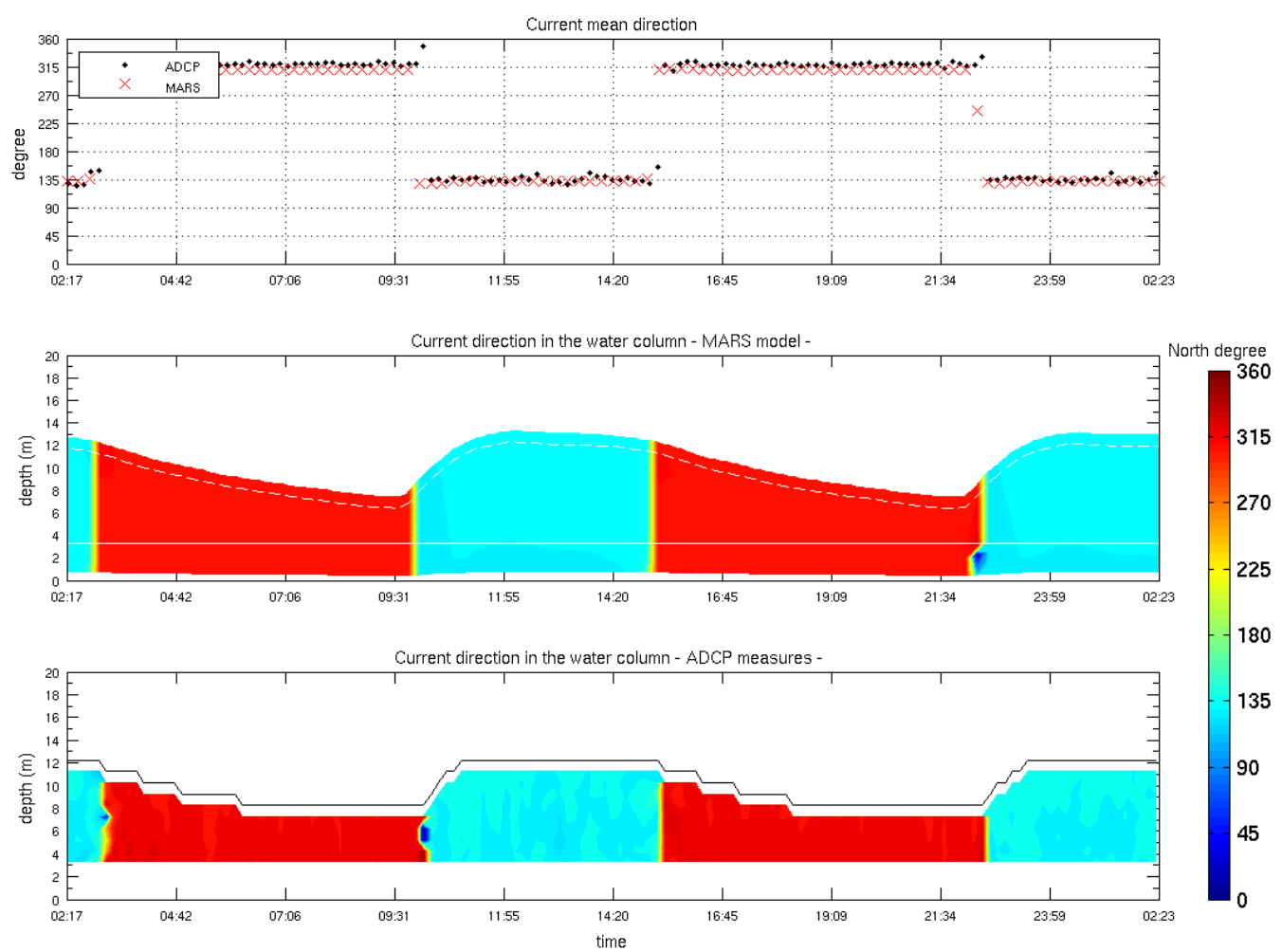

Figure 5. Direction du courant à Courval (point 2) du 13/11/2004 au 14/11/2004. 


\section{XII ${ }^{\text {èmes }}$ Journées Nationales Génie Côtier - Génie Civil \\ Cherbourg, 12-14 juin 2012}

\subsection{Salinité}

La figure 6 présente la comparaison entre les données du système de mesure en continu MAREL-Honfleur et les sorties du modèle à Honfleur en termes de salinité. Au niveau de la sortie du modèle, une moyenne des deux couches entourant le capteur du système MAREL-Honfleur (distance fixe par rapport à la surface libre) est effectuée à chaque pas de temps.

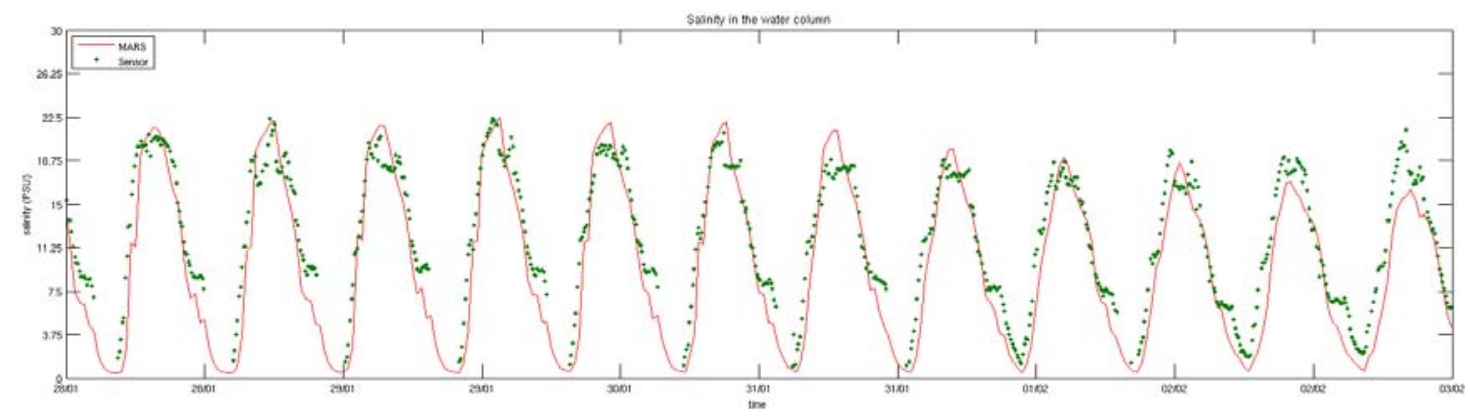

Figure 6. Comparaison de la valeur de salinité issue du modèle à l'altitude du capteur (en rouge) et des données de salinité issues du système MAREL-Honfleur (en points verts, du 28/01/2005 au 03/02/2005).

Nous voyons que le modèle reproduit de manière tout à fait satisfaisante les variations de salinité à Honfleur. En particulier, la très forte amplitude de variation au cours de la marée (de l'ordre de 20 PSU) est fidèlement simulée. Par contre le modèle ne reproduit pas le plateau de salinité révélé par les mesures pendant le jusant, dont l'origine n’a pas été expliquée.

\section{Discussion sur la comparaison aux images satellites}

Dans cette section est présentée la comparaison entre les panaches simulés et observés de la Seine en baie. Le panache simulé est représenté par la distribution des salinités de surface issues du modèle et le panache observé à travers les turbidités issues des images satellite MODIS (NASA) à 250 mètres de résolution sur l'embouchure de la Seine. Des algorithmes permettant de calculer la turbidité et les matières en suspension (PETUS et $a l ., 2010)$ ont été appliqués à ces images après avoir été calibrés (HOCER, 2011) par des données in situ récentes de l'estuaire.

Ces comparaisons sont proposées pour discuter de la forme des structures générées à l'embouchure et ne sont en aucun cas des comparaisons quantitatives, le modèle étant à l'heure actuelle uniquement hydrodynamique (transport dissous). Notons quand même que les matières en suspension générant la turbidité ambiante sont principalement issues de la rivière et leur localisation est donc liée à des valeurs de salinité faibles. D’autres comparaisons ont été testées sur les champs de température de surface, mais la faible 
résolution spatiale des images satellite empêchaient une analyse pertinente des gradients à l'échelle de l'embouchure.

La figure 7 présente les turbidités issues des images satellite et les salinités selon le modèle le 10 mars 2007 vers $13 \mathrm{~h}$, à marée haute avec un coefficient de 59. Le débit de la Seine est élevé $\left(1242 \mathrm{~m}^{3} \mathrm{~s}^{-1}\right)$ et la vitesse du vent est de $6.57 \mathrm{~m} \mathrm{~s}^{-1}$ vers l'Est.

Les structures de surface (salinité pour le modèle et turbidité pour le satellite) ont la même géométrie : turbidité élevée et eau très peu salée jusqu’à Honfleur, limite quasiméridienne entre les structures à l'embouchure, entre Le Havre et Trouville-sur-Mer.

La figure 8 présente les turbidités issues des images satellite et les salinités selon le modèle le 12 mars 2007 vers $13 \mathrm{~h}$, soit $2 \mathrm{~h}$ avant la marée haute avec un coefficient de 34. Le débit de la Seine est élevé $\left(1145 \mathrm{~m}^{3} \mathrm{~s}^{-1}\right)$ et la vitesse du vent est de $4.35 \mathrm{~m} \mathrm{~s}^{-1}$ vers le Sud-Est.

Les structures de surface (salinité pour le modèle et turbidité pour l'image satellite) ont également la même géométrie : turbidité élevée et eau très peu salée à l'intérieur des digues submersibles, sortie des panaches de forme arrondie au large du Havre.

Le modèle semble donc bien reproduire, de façon qualitative, les structures de surface observées par satellite. Une analyse quantitative sera réalisée après l'ajout d'un module sédimentaire à notre modèle (voir section suivante).

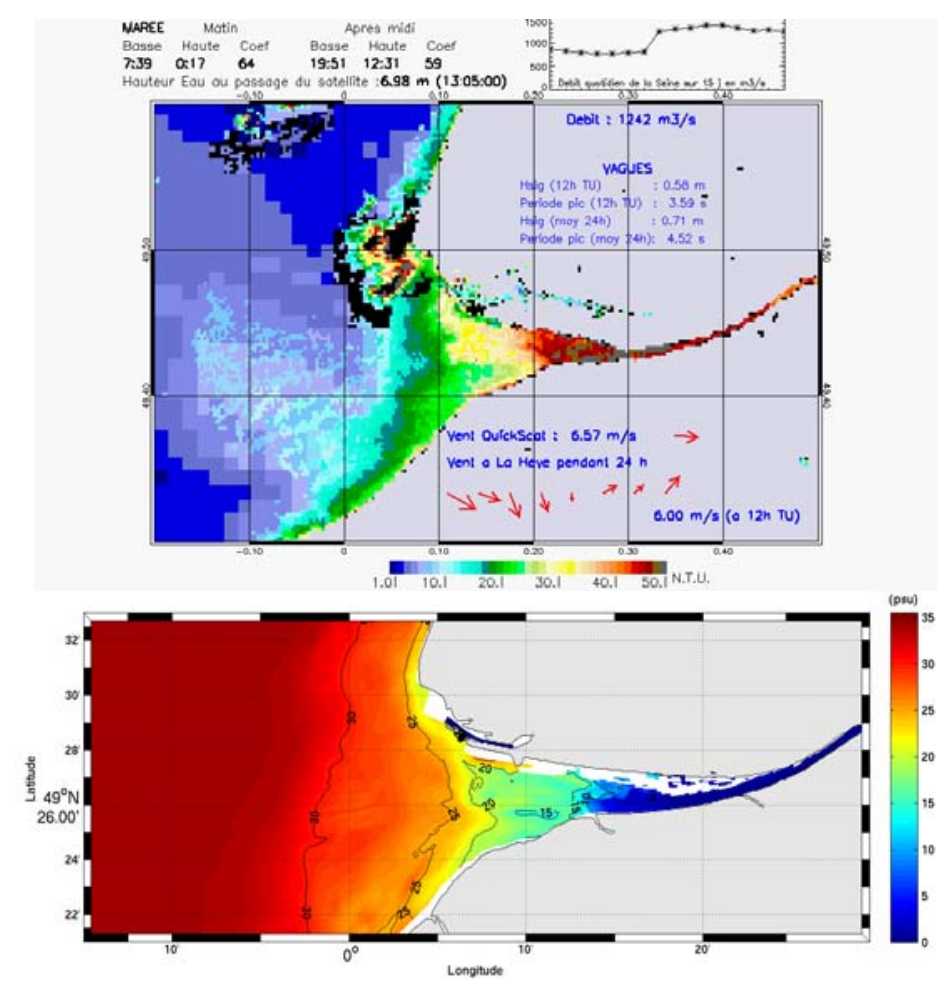

Figure 7. Comparaison qualitative du panache de la Seine le 10 mars 2007. En haut, turbidité issue de l'image satellite MODIS $250 \mathrm{~m}$. En bas, salinité de surface selon le modèle curviligne MARS3D. 


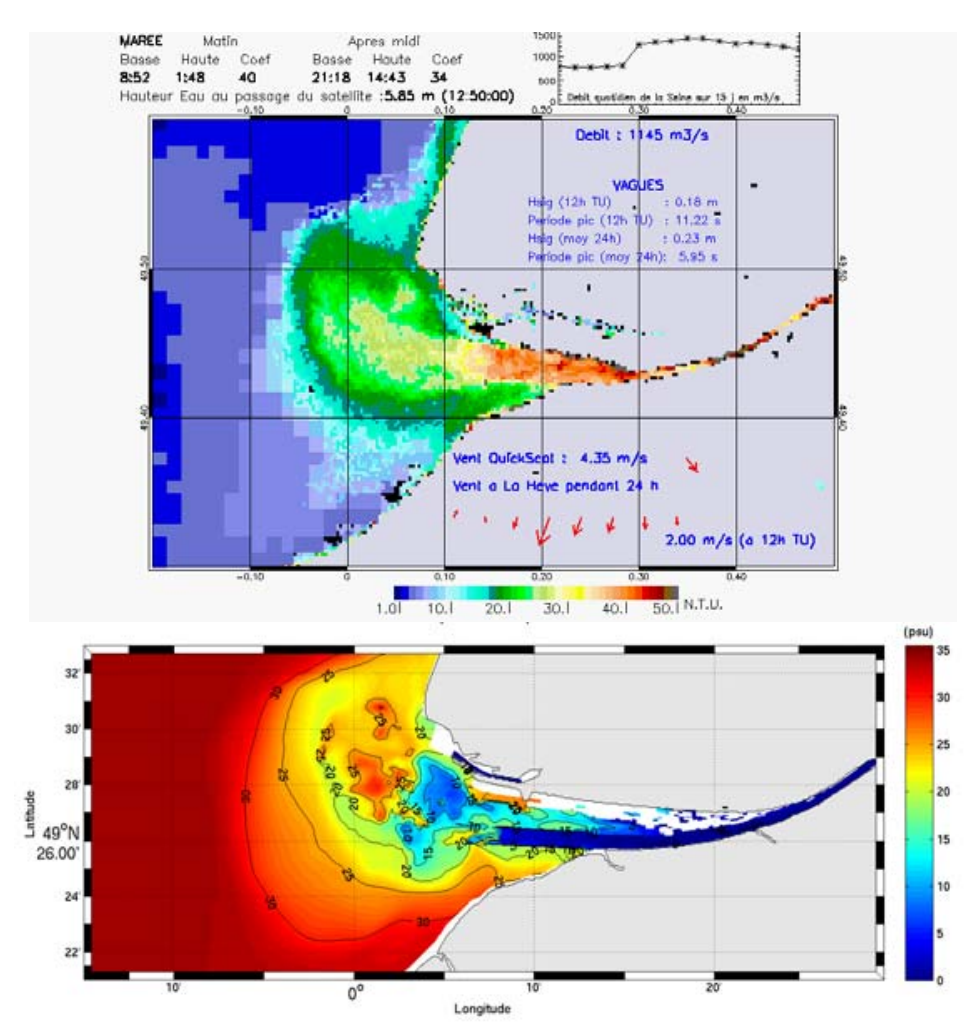

Figure 8. Comparaison qualitative du panache de la Seine le 12 mars 2007. En haut, turbidité issue de l'image satellite MODIS $250 \mathrm{~m}$. En bas, salinité de surface selon le modèle curviligne MARS3D.

\section{Conclusions et perspectives}

Les équations du modèle MARS3D ont été modifiées afin de pouvoir appliquer ce modèle aux différences finies avec un maillage à coordonnées curvilignes nonorthogonales. Grâce à ces modifications, nous avons pu raffiner le maillage aux endroits d'intérêt et modéliser finement la géométrie du fleuve et de ses méandres. Les résultats du modèle hydrodynamique sont ainsi très satisfaisants de l'amont à l'aval de l'estuaire et ce, quel que soit le débit du fleuve.

La salinité est également bien représentée à Honfleur; d'autres comparaisons (ponctuelles ou profils verticaux) devront être réalisées afin de valider ce paramètre quel que soit le débit et l'amplitude de marée.

Les variations de structures horizontales (panache) fournies en baie par le modèle ont aussi pu être validées par le panache turbide observé à l'aide du satellite MODIS (images à $250 \mathrm{~m}$ ).

Ce modèle hydrodynamique à coordonnées curviligne sera prochainement complété par un module sédimentaire (déjà inclus dans le modèle MARS3D cartésien), issu de la chaîne de modélisation SIAM (CUGIER \& LE HIR, 2002). Ce modèle multi-classes (LE HIR et al., 2011) prendra en compte les mélanges sable-vases (à l'instar de 
WAELES et al., 2008) et les processus de tassement. A cette fin, la répartition des sédiments superficiels de notre zone d'étude a déjà été analysée afin d'obtenir la répartition en cinq classes de tailles de particules du sédiment au centre de chaque maille de la grille de calcul. Ce travail s'effectue à l'Ifremer en collaboration avec le GIP Seine-Aval (LEMOINE \& GUILLERME, 2010) à partir de données géoréférencées (LESOURD, 2000).

\section{Références}

CUGIER P., LE HIR P. (2002). Development of a 3D hydrodynamic model for coastal ecosystem modelling. Application to the Plume of the Seine river (France). Estuar. Coast. And Shelf Sci. 55, pp 673-695. doi:10.1006/ecss.2001.0875

GOURRION J. (2005). Hydrodynamique bidimensionnelle en coordonnées curvilignes non-orthogonales. Rapport de post-doctorat Ifremer.

HOCER (2011). Calibration des turbidités de surface déduites des images satellite MODIS (NASA) à $250 \mathrm{~m}$ et fusion avec les images à $1 \mathrm{~km}$. Rapport final. $\mathrm{HCR} / \mathrm{BT} / 10 / 099 / \mathrm{RF}$.

LAZURE P., DUMAS, F. (2008). An external-internal mode coupling for a $3 D$ hydrodynamical model for applications at regional scale (MARS). Advances in Water Ressources, 31, pp 233-250. doi:10.1016/j.advwatres.2007.06.010

LE HIR P., CAYOCCA F., WAELES B. (2011). Dynamics of sand and mud mixtures: A multiprocess-based modelling strategy. Continental Shelf Reasearch (2011). doi:10.1016/j.csr.2010.12.009

LEMOINE J.-P., GUILLERME D. (2010). Description de la couverture sédimentaire de l'estuaire de la Seine en fonction des classes et du maillage utilisé dans le modèle curvilinéaire MARS3D Seine-Aval. Publication interne.

LESOURD S. (2000). Processus d'envasement d'un estuaire macrotidal: zoom temporel du siècle à l'heure; application à l'estuaire de la Seine. Thèse, Université de Caen, 280 p.

PETUS C., CHUST G., GOHIN F., DOXARAN D., FROIDEFOND J.M., SAGARMINAGA Y. (2010). Estimating turbidity and total suspended matter in the Adour River plume (South Bay of Biscay) using MODIS 250-m imagery. Continental Shelf Research, 30, pp 379-392. doi:10.1016/j.csr.2009.12.007

WAELES B. (2005). Modélisation morphodynamique de l'embouchure de la Seine. Thèse, Université de Caen, 230 p.

WAELES B., LE HIR P., LESUEUR P. (2008). A 3D morphodynamic process-based modelling of a mixed sand/mud coastal environment: the Seine estuary, France. In: Kusuda, T., Yamanishi, H., Spearman, J., Gailani, J.Z. (Eds), Proceedings in Marine Science. Elsevier, pp 477-498. 